07

\title{
K теории формирования крупных трещин в хрупких твердых телах
}

\author{
(C) Ю.А. Хон ${ }^{1}$, П.В. Макаров ${ }^{1,2}$ \\ ${ }^{1}$ Институт физики прочности и материаловедения СО РАН, \\ Томск, Россия \\ ${ }^{2}$ Научно-исследовательский Томский государственный университет, \\ Томск, Россия \\ E-mail: khon@ispms.tsc.ru, pvm@ispms.tsc.ru
}

Поступила в Редакцию 24 января 2021 г.

В окончательной редакции 24 марта 20201 г.

Принята к публикации 24 марта 2021 г.

\begin{abstract}
Построена модель формирования крупных трещин в хрупких твердых телах, учитывающая разные пространственно-временные масштабы накопления трещин при деформации. В рамках метода фазового поля получена система двух связанных нелинейных уравнений параболического типа, описывающих деформацию нагруженной среды при зарождении трещин на двух пространственных и временных масштабах. Найдены условия, при выполнении которых однородное распределение трещин становится неустойчивым. Развитие неустойчивости сопровождается образованием крупных трещин.
\end{abstract}

Ключевые слова: трещины, деформация, моды деформации, фазовое поле, неустойчивость.

DOI: 10.21883/FTT.2021.07.51043.014

\section{1. Введение}

Большинство природных объектов представляют хрупкие твердые тела. Их деформация под действием приложенных сил определятся зарождением и накоплением трещин и протекает на различных пространственных и временных масштабах. Процессы разрушения хрупких твердых тел на малых масштабах определяют поведение системы на больших масштабах. Экспериментальные исследования сигналов акустической эмиссии, сопровождающей образование трещин в граните, показали, что имеют место две стадии разрушения $[1,2]$. На первой стадии образующиеся трещины распределяются в объеме образца случайным образом. Зависимость напряжения от деформации практически линейна. Распределение трещин по размерам описывается нормальным распределением. На второй стадии распределение трещин по размерам имеет степенной характер. Производная $d \sigma / d \varepsilon$ меньше чем на первой стадии и уменьшается при увеличении $\varepsilon$. Здесь $\sigma-$ приложенное напряжение, $\varepsilon-$ деформация. Вторая стадия завершается образованием одной магистральной трещины либо нескольких крупных трещин, деформирующее напряжение при этом быстро падает.

Смена функции распределения от нормального распределения к степенному характерна для состояний вблизи точки фазового перехода второго рода. Зарождение и накопление трещин при деформации не относятся к фазовым превращением. Но сам факт перехода к степенному распределению трещин по размерам отражает коррелированный характер зарождения и накопления трещин на второй стадии. В отличие от фазовых переходов второго рода задача теоретического описания механизмов и кинетики локализации накопления трещин на второй стадии и образования крупных трещин не решена. Строгое решение указанной задачи предполагает решение системы уравнений, описывающих зарождение микротрещин и их взаимодействие на больших пространственных и временных масштабах и содержащих приложенное напряжение и скорость деформации в качестве управляющих параметров. Ясно, что особого оптимизма даже при записи, а тем более при решении таких уравнений испытывать не приходится, хотя работы в таком направлении имеются [3]. В этой ситуации требуются другие подходы к ее решению. Один из таких подходов изложен ниже.

\section{2. Модель деформируемого твердого тела}

Рассматривается хрупкая случайно-неоднородная среда, которая деформируется в условиях постоянного всестороннего сжатия и одноосного осевого нагружения $\sigma$ вдоль оси $x$ с постоянной скоростью деформации $\dot{\varepsilon}$. В дальнейшем рассматривается одномерный случай. Петрографические особенности и хрупкопластичность среды не рассматриваются. На всех стадиях макроскопическая необратимая деформация определяется зарождением трещин с различными характерными размерами и сопровождается понижением упругой энергии среды. Площадь трещины пропорциональна квадрату ее характерного размера. Механизм и динамика зарождения отдельных трещин не рассматриваются. На первой стадии корреляция между процессами зарождения трещин отсутствует. В случайно-неоднородной среде их распределение по размерам подчиняется нормальному распределению. При увеличении напряжения число трещин увеличивается, среднее расстояние между ними уменьшается. При достижении порогового значения плотности трещин (напряжения) образование трещин протекает 
коррелированным образом и сопровождается образованием трещин с большим характерным размером. Это отражается в степенном распределении числа трещин по размерам на второй стадии. Обозначим через $l_{1}$ характерный размер области, в которой процессы образования трещин коррелированы. При этом $L \gg l_{1} \gg l_{0}$. Здесь $L$ - длина образца, $l_{0}$ - среднее расстояние между трещинами. Локальную деформацию на этом масштабе обозначим через $\varepsilon_{1}(x, t)$ и для определенности назовем ее первичной модой деформации. Здесь время $t=\frac{\varepsilon}{\dot{\varepsilon}}$.

Образование крупных трещин, предшествующих формированию магистральной трещины, отражает коррелированное смещения объемов среды на масштабах $L>l_{2} \gg l_{1}$. Деформацию $\varepsilon_{2}(x, t)$, определяемую процессами на масштабе $l_{2}$, назовем вторичной модой деформации. В каждый момент времени проявляются те моды деформации, при которых упругая энергия среды при заданном напряжении и заданной скорости деформации понижается за меньшее время.

Для математического описания процессов деформации на разных масштабах используется метод фазового поля [4]. Под фазовым полем понимаются величины, количественно характеризующие качественные изменения в системе. В теории фазовых переходов указанные переменные, введенные Ландау, называются параметрами порядка. Качественные изменения в хрупкой среде определяются зарождением трещин. Поэтому в качестве переменной на масштабе $l_{1}$ выбрана локальная концентрация образовавшихся трещин $n(x, t)=\frac{N(x, t)}{N(t)}$. Здесь $N(x, t)$ - число трещин в единице объема, $N(t)-$ общее число трещин. Локальную деформацию $\varepsilon_{1}(x, t)$, определяемую локальной концентрацией образовавшихся трещин $n(x, t)$, представим в виде

$$
\varepsilon_{1}(x, t)=\bar{\varepsilon}_{1} n(x, t) .
$$

Здесь $\bar{\varepsilon}_{1}$ - параметр, определяемый свойствами среды и образующихся трещин. Удобство введения переменной $n(x, t)$ состоит в том, что она, во-первых, является скалярной величиной. Во-вторых, явно не зависит от механизма образования трещин. И, в-третьих, $0 \leq n \leq 1$, т.е. является малым параметром. Характерное время изменения $n$ обозначим через $t_{1}$. При этом $t_{1} \ll t$. Вторичную моду деформации определим выражением

$$
\varepsilon_{2}(x, t)=A(x, t) \bar{\varepsilon}_{2} .
$$

Здесь $\bar{\varepsilon}_{2}$ - параметр, определяемый свойствами среды. Переменная $A(x, t)$ имеет смысл локальной концентрации трещин, определяющих вторичную моду деформации. Величина $t_{2}$ определяет характерное время изменения $A$. Из определения $A$ следует, что $0 \leq A<1$. С точки зрения теории систем, далеких от состояния равновесия [5-7], переменные $n(x, t), A(x, t)$ представляют амплитуды неустойчивых мод, возбуждающихся вдали от состояния равновесия.

Общая локальная деформация среды $\varepsilon(x, t)$ представляет сумму двух мод деформации

$$
\varepsilon(x, t)=n(x, t) \bar{\varepsilon}_{1}+A(x, t) \bar{\varepsilon}_{2},
$$

а скорость деформации

$$
\dot{\varepsilon}(x, t)=\dot{n}(x, t) \bar{\varepsilon}_{1}+\dot{A}(x, t) \bar{\varepsilon}_{2} .
$$

Из кинематических соображений следует, что

$$
\left\langle\dot{n}(x, t) \bar{\varepsilon}_{1}+\dot{A}(x, t) \bar{\varepsilon}_{2}\right\rangle>\dot{\varepsilon} .
$$

Угловые скобки означают усреднение по длине. По существу, условие (5) выделяет те моды деформации, которые приводят к релаксации упругой энергии среды при заданной скорости изменения нагрузки.

Для нахождения кинетических уравнений для амплитуд в методе фазового поля используются известные подходы Гинзбурга-Ландау и Ландау-Халатникова. Для деформируемой среды функционалом Гинзбурга-Ландау является потенциальная энергия системы. Первичная мода деформации возбуждается, когда упругая энергия системы при образовании трещин понижается. Разложение плотности потенциальной энергии $u_{1}(n, \sigma)$ по степеням $n$ и градиентам $n$ имеет вид

$$
\begin{aligned}
& u_{1}(n, \sigma)=u(\sigma) \\
& \quad+\left[-\frac{\alpha n^{2}}{2}-\frac{q_{2} n^{3}}{3}+\frac{q_{3} A n^{4}}{4}+\frac{q A n^{2}}{2}+\frac{g(\nabla n)^{2}}{2}+\ldots\right] .
\end{aligned}
$$

Здесь $\alpha(\sigma), q_{2}(\sigma)>0, q_{3}(\sigma)>0, g>0$ представляют коэффициенты разложения, определяемые свойствами деформируемой среды. Параметр $\alpha$ может иметь разный знак. Второе слагаемое в квадратных скобках означает, что имеется потенциальный барьер, разделяющий два состояния (бистабильность) среды. Знак „ $+{ }^{“}$ перед коэффициентом $q$ в правой части (6) означает, что возбуждение вторичной моды повышает потенциальную энергию системы. Принимая во внимание (6), уравнение Ландау-Халатникова имеет вид

$$
t_{1} \partial_{t} n=[\alpha(\sigma)-q(\sigma) A] n+q_{2} n^{2}-q_{3} n^{3}+l_{1}^{2} \partial_{x^{2}}^{2} n .
$$

Возбуждение вторичной моды протекает на фоне первичной моды и происходит гомогенно (отсутствует потенциальный барьер, разделяющий два состояния). В этом случае разложение потенциальной энергии $u_{2}$ имеет вид

$$
\begin{aligned}
u_{2}(A, \sigma) & =u_{2}(\sigma) \\
& +\left[\frac{a A^{2}}{2}+\frac{b n^{4}}{4}-\frac{p n A^{2}}{2}+\frac{f(\nabla n)^{2}}{2}+\ldots\right] .
\end{aligned}
$$

Здесь $a(\sigma)>0, b(\sigma)>0, p(\sigma)>0, f>0$ коэффициенты разложения, определяемые свойствами среды. Знак „-“ перед $p$ в правой части (8) означает, что возбуждение первичной моды инициирует вторичную моду деформации (потенциальная энергия понижается). При $a>0$, потенциальная энергия повышается. Уравнение Ландау-Халатникова имеет вид

$$
t_{2} \partial_{t} A=(-a+p n) A-b|A|^{2} A+l_{2}^{2} \partial_{x}^{2} A .
$$


Уравнение (9) при $p=0$ и $a>0$ имеет единственное устойчивое решение $A_{0}=A=0$. Вторичная мода не возбуждается. Это означает, что зарождение вторичной моды деформации определяется первичной модой деформации. Коэффициент $a(\sigma)$ уменьшается с увеличением $\sigma$. К уравнениям (7), (9) следовало бы добавить уравнение, связывающее приложенное напряжение с величиной деформации. В настоящей работе предполагается, что такая связь известна из эксперимента.

Замена переменных

$$
\begin{aligned}
& \tilde{t}=|a| t / t_{2}, \quad \tilde{x}=|a|^{1 / 2} x / l_{2}, \\
& \tilde{A}=A b^{1 / 2}|a|^{-1 / 2}, \quad \tilde{n}=n q_{3}^{1 / 2}
\end{aligned}
$$

приводит уравнения (7), (9) к виду (знак „ “ в дальнейшем опускается)

$$
\begin{gathered}
\partial_{t} A=(-1+d n) A-A^{3}+\partial_{x}^{2} A, \\
\tau \partial_{t} n=(\alpha-c A) n+\beta n^{2}-n^{3}+l^{2} \partial_{x}^{2} n .
\end{gathered}
$$

Здесь

$$
\begin{aligned}
& \tau=|a| \frac{t_{1}}{t_{2}}, \quad l=|a|^{1 / 2} \frac{l_{1}}{l_{2}}, \quad \beta=q_{1} / q_{3}^{1 / 2}, \\
& d=p_{1} /\left(|a| q_{3}^{-1 / 2}\right), \quad c=q\left(\frac{|a|}{b}\right)^{1 / 2} .
\end{aligned}
$$

Уравнения (11), (12) представляют систему нелинейных параболических уравнений, описывающих эволюцию деформируемой среды при зарождении трещин на двух масштабах. Управляющим параметром в этих уравнениях является приложенное напряжение $\sigma$, от которого зависят все коэффициенты в уравнениях (13). Постоянство $\sigma$ означает, что рассматриваются релаксационные процессы, скорости которых намного превышают скорости деформации образца.

\section{3. Однородные стационарные решения и их устойчивость}

Уравнения (11), (12) всегда имеют однородное стационарное решение $A_{0}=A=0, n_{0}=n=0$. При $\alpha<-\frac{\beta^{2}}{4}$ это решение единственно, что имеет место на первой стадии деформации. Однородное стационарное решение $A=0, \quad n_{h}=\frac{\beta}{2}+\left(\frac{\beta^{2}}{4}+\alpha\right)^{1 / 2}$ стабильно при $-\frac{2 \beta^{2}}{9}<\alpha<0$ и метастабильно при $-\frac{\beta^{2}}{2}<\alpha<-\frac{2 \beta^{2}}{9}$. При $\alpha=-\frac{2 \beta^{2}}{9}$ оба решения имеют одинаковую устойчивость. Учитывая зависимость $\alpha, \beta$ от напряжения, уравнение $-\frac{2 \beta^{2}}{9}=\alpha$ определяет пороговое значение напряжения, выше которого имеющиеся в среде неоднородности могут инициировать зарождение трещин на второй стадии. Третье решение $A=0, n_{u}=\frac{\beta}{2}-\left(\frac{\beta^{2}}{4}+\alpha\right)^{1 / 2}$ всегда неустойчиво и определяет максимум потенциальной энергии (6). Для перехода через потенциальный барьер

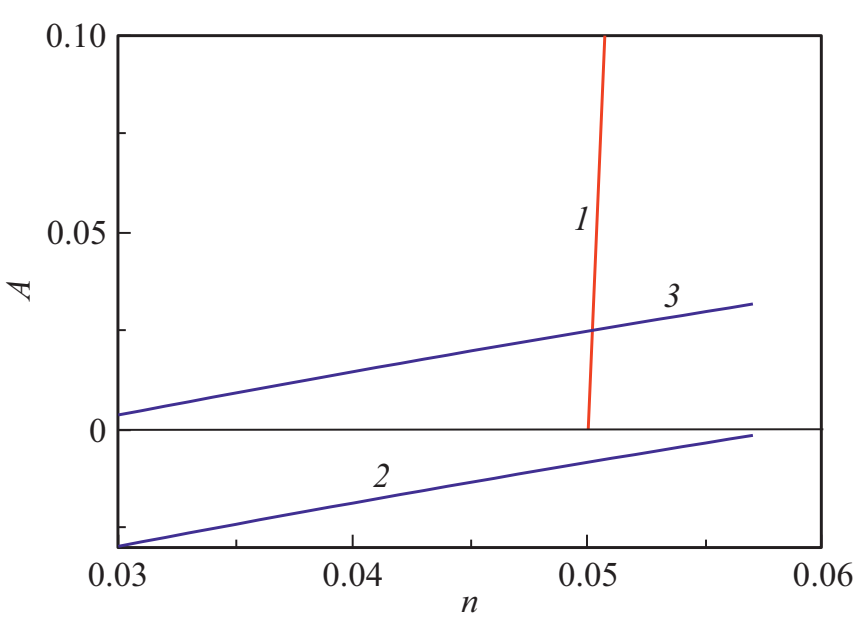

Рис. 1. Нуль-изоклины уравнений (12) (кривая 1) и (11) (кривые $2,3,4)$ при $\alpha(2)<\alpha(1)$. Цифры в скобках у $\alpha$ указывают номер кривой.

амплитуда начального возмущения должна превышать величину $n_{u}$. При $\alpha>0$ решение $n_{h}$ стабильно, любое малое возмущение $n$ нарастает. Трещины спонтанно не зарождаются, поэтому при всех напряжениях $\alpha<0$. Однородные стационарные решения $A_{s}>0, n_{s}>0$ уравнений (11), (12) определяются точками пересечения кривых

$$
\begin{gathered}
H=-1+d n-A^{2}=0, \\
\Phi=\alpha-c A+\beta n-n^{2}=0 .
\end{gathered}
$$

Из уравнений (14), (15) следует, что такие решения существует при выполнении неравенств

$$
\begin{gathered}
-1+d(\sigma) n>0, \\
-\frac{2 \beta^{2}}{9}<\alpha<0 .
\end{gathered}
$$

Неравенство (17) означает, что решение $A_{0}, n_{s}>0$ возбуждается при начальном возмущении $\Delta n$, превышающем пороговое значение $n_{u}$. Деформация при разрушении образца из гранита не превышает 2-3 процентов [1]. Поэтому интерес представляют решения $A_{s} \ll 1, n_{s} \ll 1$. Как следует из анализа уравнений (14), (15), такие решения существуют при

$$
-\beta / \alpha>d(\sigma)>1 / n
$$

При $n_{s} \approx 0.05 d(\sigma) \approx 20$. В качестве примера на рис. 1 приведены зависимости $A=A(n)$ при двух значениях $\alpha(\sigma)=-0.02, \alpha(\sigma)=-0.01$ и постоянных значениях $d=15, \beta=0.4, c=0.3$ в уравнениях (14), (15). Видно, что решение $A_{s} \ll 1, n_{s} \ll 1$ существует при $\alpha=-0.01$ и не существует при $\alpha=-0.02$. Это означает наличие порогового значения напряжения для перехода ко второй стадии деформации. При напряжении, меньшем порогового значения, зарождаются отдельные трещины на масштабах, меньших $l_{1}$. Их зарождение происходит при 
увеличении приложенного напряжения, что отражается в зависимости параметров в уравнениях (11), (12) от напряжения.

Стандартный анализ устойчивости решения $A_{s}, n_{s}$ показывает, что оно устойчиво относительно малых однородных возмущений, но неустойчиво относительно неоднородных возмущений конечной амплитуды при

$$
\tau<1, \quad l \ll 1, \quad \tau>l .
$$

С учетом (13), неравенства (19) принимают вид

$$
\frac{l_{1}}{l_{2}} \ll 1, \quad \frac{t_{1}}{t_{2}}<1
$$

Отсюда следует, что характерные скорости $v_{A}=\frac{l_{2}}{t_{2}}$, $v_{n}=\frac{l_{1}}{t_{1}}$ связаны неравенством $v_{A} \gg v_{n}$. Первое неравенство означает, что локализация разрушения, определяется процессами релаксации, протекающими на двух различающихся масштабах. При этом на меньшем масштабе время релаксации меньше (второе неравенство). При выполнении неравенств (20) развитие неустойчивости приводит к локализации деформации, формированию и развитию очага разрушения.

\section{4. Зарождение и развитие крупной трещины}

Неоднородные локализованные решения $n(x, t)$, $A(x, t)$ описывающие зарождение и развитие крупной трещины, получены численным решением системы уравнений (11), (12) в интервале $0 \leq x \leq X \ll L$. При $t=0$ система находится в состоянии $A_{0}, n_{0}$ в конце первой стадии. Решение $A_{0}, n_{0}$ неустойчиво относительно возмущений конечной амплитуды. Начальное возмущение для переменной $n$ задано в виде $\Delta n=\Delta n_{0} \exp \left[-\sigma_{n}\left(x-x_{0}\right)^{2}\right]$. Здесь $\Delta n_{0}, \sigma_{n}$, $x_{0}$ - амплитуда, дисперсия и координата начального возмущения соответственно. Рассматривается динамика зарождения и развития одной крупной трещины. То есть предполагается, что крупные трещины находятся далеко друг от друга. Начальное возмущение для переменной $A$ взято стохастическим с амплитудой $0 \leq \Delta A(x) \leq 10^{-3}$. Задавались периодические граничные условия. Ниже приводятся результаты для

$$
\begin{gathered}
d=15, \quad \alpha=-0.01, \quad \beta=0.4, \\
c=0.3, \quad \tau=0.8
\end{gathered}
$$

и разных $l$. Это позволяет проследить роль масштабного фактора. При выбранных значениях параметров в (21) $A_{s} \approx 0.04, n_{s} \approx 0.07$. Параметры начального возмущения для $n$ :

$$
\Delta n_{0}=0.05, \quad \sigma_{n}=3, \quad x_{0}=20 .
$$

На рис. 2 приведены пространственные распределения переменных $n, A$ в разные моменты времени при $l=0.1$.

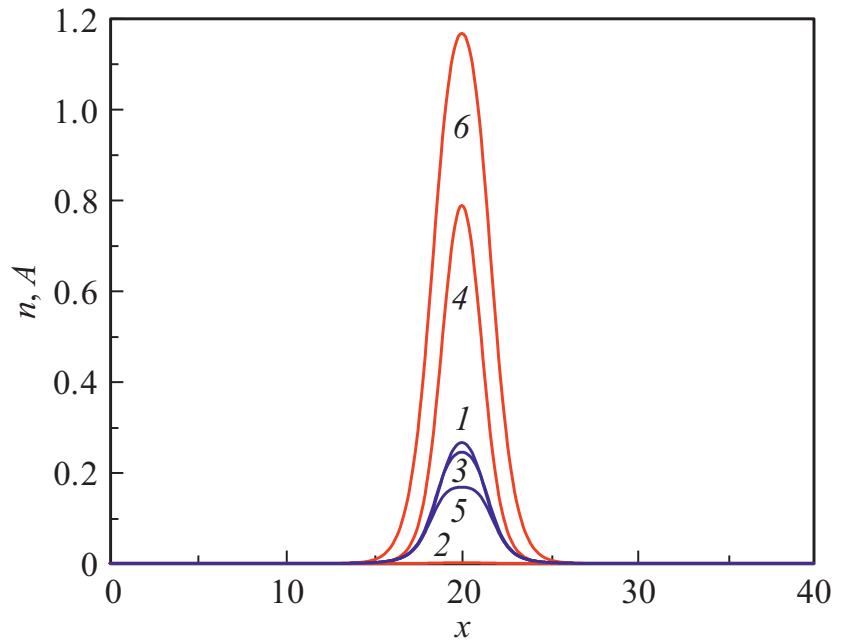

Рис. 2. Распределение переменных $n$ (кривые $1,3,5$ ) и $A$ (кривые 2, 4,6) в моменты времени $t=111$ (кривые 1,2), $t=113$ (кривые 3,4), $t=115$ (кривые 5,6).

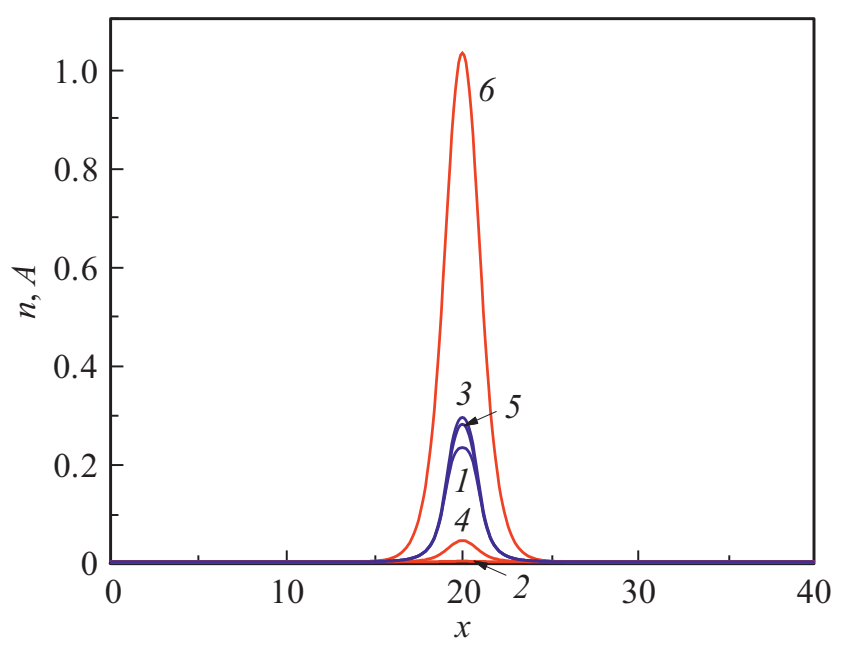

Рис. 3. Распределение переменных $n$ (кривые $1,3,5$ ) и $A$ (кривые 2, 4,6) в моменты времени $t=77$ (кривые 1,2 ), $t=79$ (кривые 3,4$), t=81$ (кривые 5, 6).

При этом значении $l v_{A}=8 v_{n}$. Видно, что имеются два этапа. На первом этапе переменная $n$ нарастает до значения $n \approx 0.27$ за время $t \approx 111$. Обращает на себя внимание локализованный характер решения. Это решение описывает локализованное накопление трещин, характерное для второй стадии деформации. Переменная $A$ на этом этапе близка к нулю. Когда величина $(-1+d \varphi)$ становится положительной, наступает второй этап. Переменная $A$ быстро возрастает и к моменту времени $t=115$ достигает максимального значения $A_{m} \approx 1.2$. При этом $n$ уменьшается до значения $n \approx 0.17$. Скорость нарастания переменной определяется величиной $(-1+d \varphi)$. Это видно из уравнения (11), в котором $\dot{A} \sim(-a+d \varphi) A$. Быстрый рост переменных означает высокую скорость деформации. Эксперимен- 


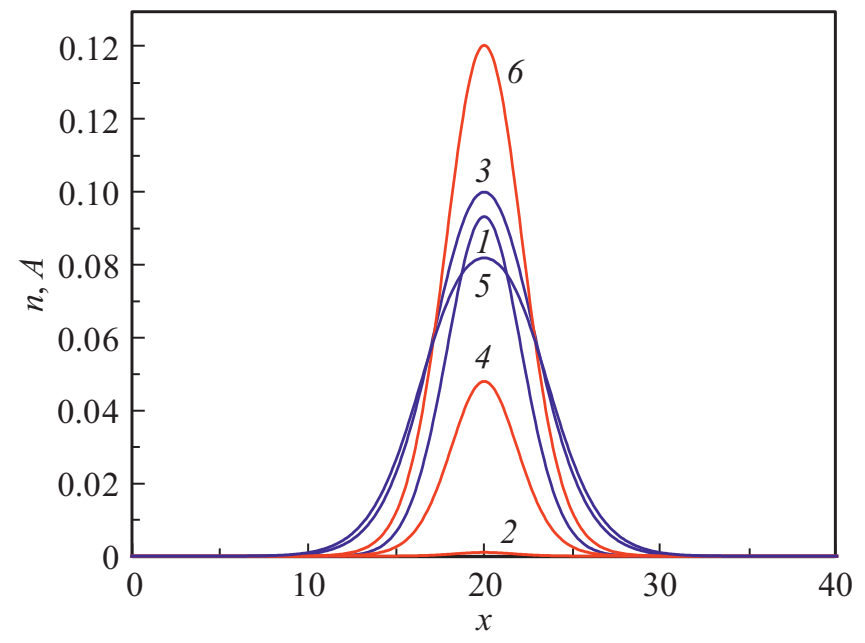

Рис. 4. Распределение переменных $n$ (кривые 1,3,5) и $A$ (кривые $2,4,6)$ в моменты времени $t=10$ (кривые 1,2 ), $t=25$ (кривые 3,4$), t=32$ (кривые 5, 6).

тально это проявляется в быстром падении величины деформирующего напряжения (при постоянной скорости деформации) в конце второй стадии при формировании системы крупных трещин на большом пространственном масштабе. Математически рассмотренные локализованные решения представляют статические автосолитоны и описывают неравновесные состояния деформируемой среды [8].

На рис. 3 приведены пространственные распределения переменных $n, A$ при значении $l=1 / 50$. Видно, что динамика формирования крупной трещины качественно не изменилась. Уменьшилась амплитуда переменной $A$ и ширина области локализации переменной $n$, изменилось время локализации.

Пространственные распределения переменных $n, A$ при значении $l=0.3$ и $\Delta n_{0}=0.1$ приведены на рис. 4 . В этом случае начальное возмущение $\Delta n_{0}$ должно превышать величину $\approx 0.9$. Видно, что ширина областей локализации переменных $A$ и $n$ стали близкими, амплитуды переменных стали меньше таковых, приведенных на рис. 2,3. Время нарастания переменной $A$ до максимального значения увеличилось примерно в пять раз. При $l=0.3$ локализация не происходит.

\section{5. Заключение}

В предложенной модели формирования и развития крупной трещины в хрупком твердом теле, деформируемом с постоянной скоростью, ключевая роль отводится нелинейной динамике двух мод деформации. Каждая мода определяет релаксацию упругой энергии среды и характеризуется пространственным и временным масштабом коррелированного зарождения и накопления трещин. Отношение пространственного масштаба к вре- менно́му определяет характерную скорость релаксации упругой энергии системы.

Локализованное накопление трещин, формирование и развитие крупной трещины в среде определяется возбуждением крупномасштабной пространственной моды с высокой скоростью релаксации упругой энергии. В твердом теле, деформируемом в условиях всестороннего сжатия и одноосного осевого нагружения, данная мода проявляется в виде зарождения крупных трещин, предшествующих образованию магистральной трещины. Если исключить возбуждение указанной крупномасштабной пространственной моды, то формирование и развитие крупной трещины может быть предотвращено.

\section{Благодарности}

Авторы благодарят Л.Б. Зуева за полезные советы и обсуждение результатов.

\section{Финансирование работы}

Исследование выполнено за счет гранта Российского научного фонда (проект № 19-17-00122).

\section{Конфликт интересов}

Авторы заявляют, что у них нет конфликта интересов.

\section{Список литературы}

[1] А.Г. Кадомцев, Е.Е. Дамаскинская, В.С. Куксенко. ФТТ 53, 1777 (2011)

[2] Е.Е. Дамаскинская, А.Г. Кадомцев. Письма в ЖТФ 39, 29 (2013).

[3] П.В. Макаров, И.Ю. Смолин, А.Ю. Перышкин, А.С. Кульков, Р.А. Бакеев. Физическая мезомеханика 23, 5, 43 (2020).

[4] Ю.А. Хон, Л.Б. Зуев. ФТТ 62, 2020 (2020).

[5] M.C. Cross, P.C. Hohenberg. Rev. Mod. Phys. 65, 854 (1993).

[6] I.S. Aranson. Rev. Mod. Phys. 74, 99 (2002).

[7] P.C. Hohenberg, A.P. Krekhov. Phys. Rep. 572, 1 (2015).

[8] Б.С. Кернер, В.В. Осипов. УФН 157, 201 (1989).

Редактор Т.Н. Василевская 